\title{
GD1212: Probing deep into the interior of a pulsating white dwarf star
}

\author{
N. Giammichele ${ }^{1, a}$, G. Fontaine ${ }^{1}$, S. Charpinet ${ }^{2}$, P. Brassard ${ }^{1}$, and S. Greiss ${ }^{3}$ \\ 1 Université de Montréal, Montréal, Québec, Canada \\ 2 Institut de Recherche en Astrophysique et Planétologie (IRAP), Observatoire Midi-Pyrénées, Uni- \\ versité Paul Sabatier / CNRS, Toulouse, France \\ 3 The University of Warwick, Coventry CV4 7AL, UK
}

\begin{abstract}
We present the first self-consistent seismic analysis of a white dwarf star, GD 1212, in the Kepler 2 field. We precisely establish the fundamental parameters of the star using the forward method based on physically sound models. We unravel the internal structure as well as the rotation profile of GD1212 deeper than in any other ZZCeti stars studied so far. This opens up interesting prospects for future analyses of the white dwarf pulsators monitored in the Kepler and Kepler 2 fields.
\end{abstract}

\section{Introduction}

Efforts made on the search for pulsating white dwarfs in the Kepler field have proven to be quite successful with the discovery of several new ZZ Ceti stars. The analysis presented here focus on the ZZ Ceti star: GD 1212, which was observed by Kepler-2 in the test run field (see [4] for more details). GD 1212 was specifically picked as it represents the boundary of the pure ZZ Ceti instability strip. Spectroscopic determination of $\log g$ and $T_{\text {eff }}$ places GD 1212 near the red edge. This is further confirmed by the comparison between their mean excited period and their position in the $\log g-T_{\text {eff }}$ diagram as presented in Figure 1.

The light curve of GD 1212 is composed of 9 consecutive days of data. It reveals a quite complicated structure, standard for stars further away from the blue edge, where the simple pulsators are usually located. As we move along the cooling track of WDs, cooler ZZ Ceti stars have pulsation spectra with numerous harmonics, frequency sums and differences associate with nonlinear pulse shapes. Pulsation spectra can furthermore vary importantly with time, which tends to make the mode identification exercise quite challenging. Standard reduction techniques were used on GD 1212 light curve; details can be found in [4]. The spectrum of GD 1212 is quite rich as we can use thirteen independent modes for the internal structure of the star, and nineteen components of rotationally-split multiplets for the internal rotation profile.

\section{Internal structure}

The technique relies on a double-optimization scheme that, first, best matches the observed periods with the periods calculated from a theoretical, parameterized model, using a $\chi^{2}$ formalism. The second optimization uses a multimodal optimizer based on a genetic algorithm to find the best-fit model in parameter space, the quality of the fit being quantified by a merit function. We search around a very large domain around the spectroscopic values to guide us. It is important to point out that there is no a priori guaranty to find an optimal model that could match the spectroscopic solution. The onion-like

\footnotetext{
a e-mail: noemi@astro.umontreal.ca
}

This is an Open Access article distributed under the terms of the Creative Commons Attribution License 4.0, which permits unrestricted use, distribution, and reproduction in any medium, provided the original work is properly cited. 


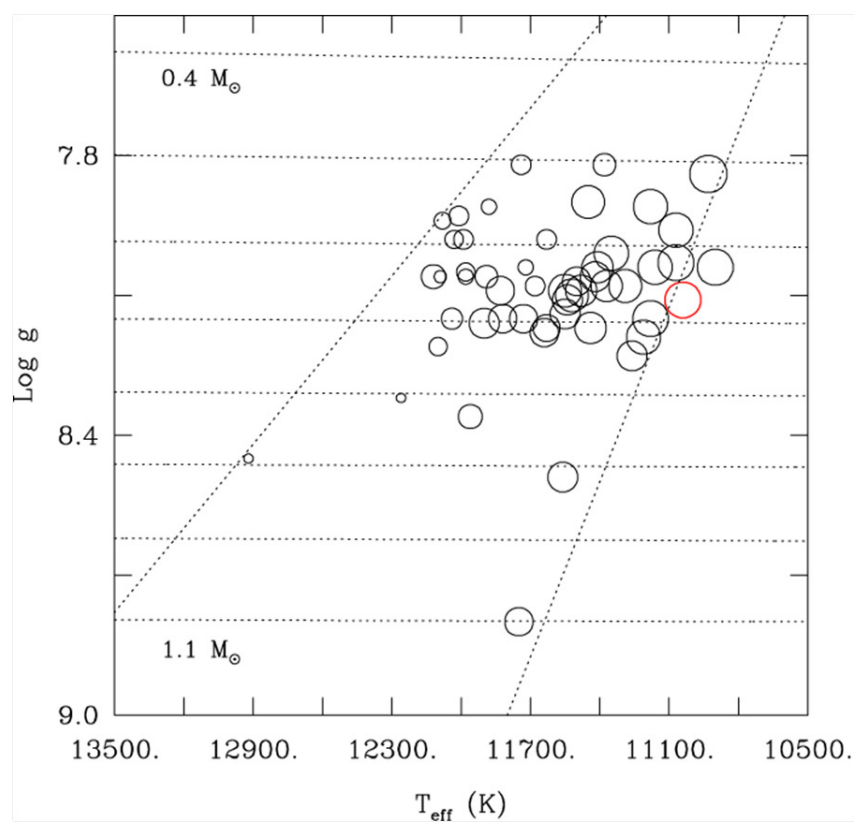

Fig. 1. Correlation between excited period and effective temperature or surface gravity for ZZ Ceti stars. The size of the open circles gives a measure of the representative period on a logarithmic scale for each star. The two diagonal dotted lines are the empirical blue and red edge of the instability strip. Figure originating from [2].

structure of the white dwarf is best described by a parameterized model with eight parameters: $T_{\text {eff }}$, $\log g, D(H), D(H e), P f_{1}, P f_{2}$, (uniform) core composition and convective efficiency. $P f_{1}$ and $P f_{2}$ are inherent to the chemical layering of the two outermost layers, and represent the steepness of the chemical transition between the $\mathrm{H} / \mathrm{He}$ layers and the $\mathrm{He} / \mathrm{CO}$ core, respectively. Tests have shown that for low radial order, models are quite insensitive to the choice of the mixing length theory. Standard value of ML2 $/ \alpha=1.0$ is then fixed in our case. The pulsation periods detected in ZZ Ceti pulsators are mostly sensitive to the first four parameters: surface gravity, effective temperature, and envelope layering, particularly the thickness of the outermost hydrogen envelope. But there is also some sensitivity of the pulsation periods to the core composition, more difficult to exploit since we must rely on the detection of modes that have relatively large amplitudes in the core.

Figure $2 \mathrm{a}$ shows the projection on the $T_{\text {eff }}-\log g$ plane of the merit function for GD 1212. The logarithmic scale emphasizes the great contrast in $S^{2}$ between the minimum solution (blue) and maxima located elsewhere in parameter space (red). In both cases, we converge toward a particular solution that fits nicely within the spectroscopic solution bounding box. Resulting parameters are presented in the following table:

\begin{tabular}{|c|c|c|c|c|c|c|}
\hline & $T_{\text {eff }}$ & Spect. $T_{\text {eff }}$ & $\log g$ & Spect. $\log g$ & $\mathrm{D}(\mathrm{H})$ & $\mathrm{D}(\mathrm{He})$ \\
\hline \hline GD 1212 & 11,244 & 11,035 & 8.10 & 8.08 & -6.04 & -2.82 \\
\hline
\end{tabular}

The case of the use of non-parameterized $\mathrm{C} / \mathrm{O}$ core descriptions coming from evolutionary sequences, has to be dealt with great precaution. White dwarf models derived from main sequence evolution suffer from many uncertainties and are numerically too noisy to use for quantitative adiabatic asteroseismology. We can see an example of such an experiment in the case of GD1212 in Figure 2b. Not only the best $\mathrm{S}^{2}$ is noticeably degraded from 28 to 252 , the mapping of the solution on the $T_{\text {eff }}-\log$ $g$ plane is scattered with local minima due to the numerical noise from the evolutionary profile. The best fit does not even lie within the spectroscopic limits anymore. Similar experiment with other ZZ Ceti stars yelds similar results. Parameterized models are much better suited for this purpose.

If we look at Figure 3a, we can see the weight functions of the different modes present in the pulsation spectrum. Evolution has pushed the g-modes to the outside because of the increase of de- 

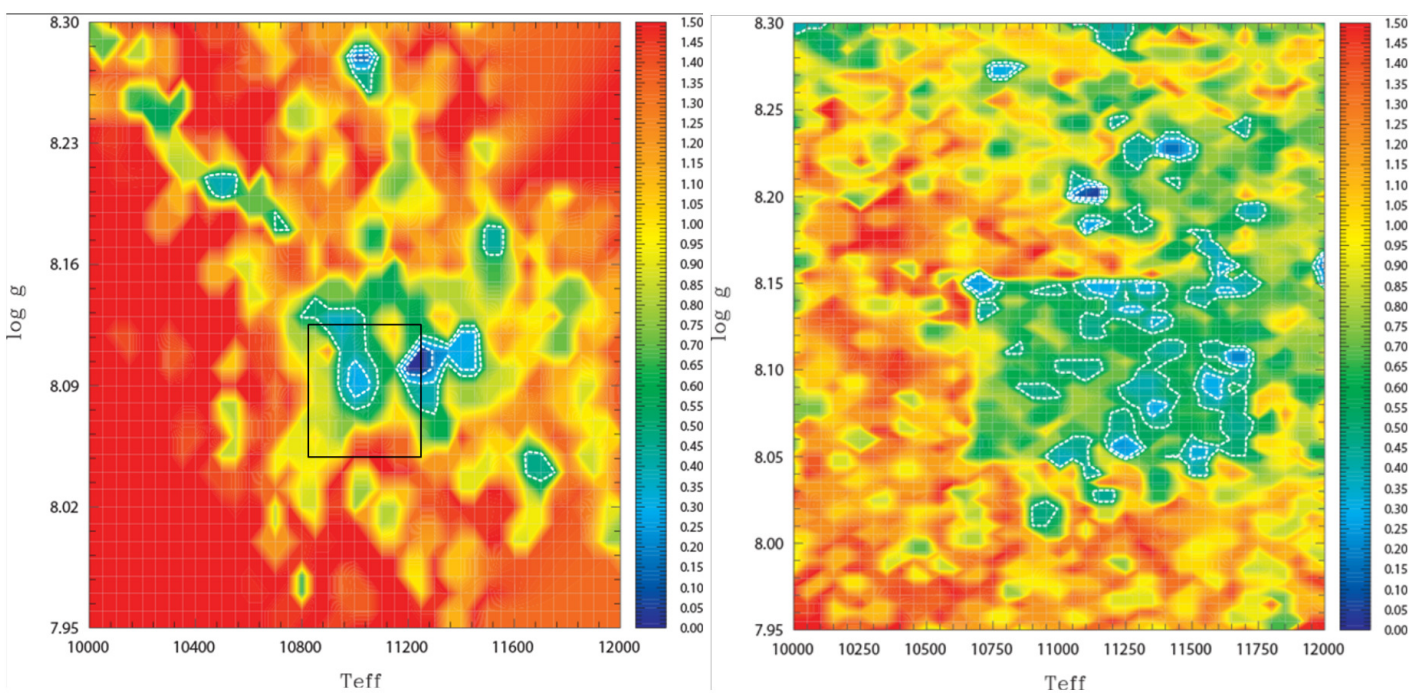

Fig. 2. Projection on the $T_{\text {eff }}-\log g$ plane of the merit function $S^{2}$ for GD 1212. The spectroscopic solution given within $1 \sigma$ is illustrated with a black box.Left: Homogeneous C/O core. Right: Non-homogeneous core with chemical profiles coming from evolutionary models.
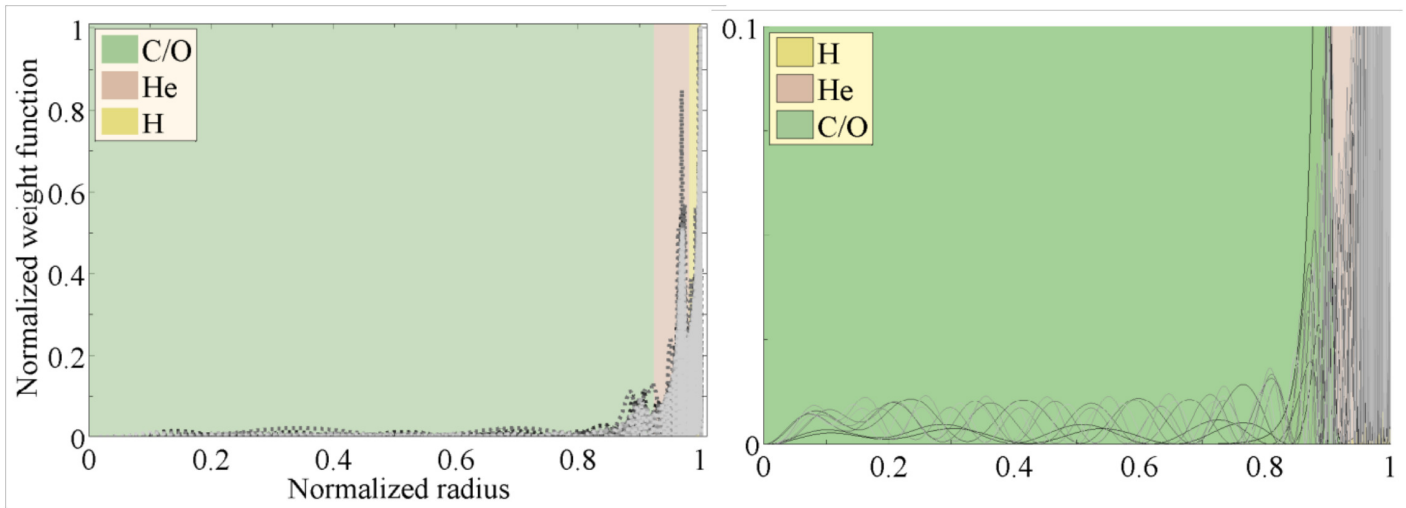

Fig. 3. Left: Normalized weight functions of the modes present in GD 1212 as a function of the radius of the star. The area under the each curve is the same. Right: Zoom-in view of the normalized rotation kernels as a function of radius for each mode identified in the optimal model of GD 1212.

generacy along the cooling track. This implies as we see in Figure 3a that weight functions have large amplitude in the outermost layers. But we can also notice that the core is almost entirely sampled by low-amplitude weight functions. Taking advantage of this behavior, we can properly assess the bulk $\mathrm{C} / \mathrm{O}$ core composition to be $47-53 \%$. Since the interior of the star is not entirely sampled, it is important in order to infer the proper bulk composition of the star to have some kind of normalization. If we consider a profile coming from evolutionary sequences at the same effective temperature and similar surface gravity, by normalizing with the weight functions we can compare directly the two compositions. By doing so, we find quite a nice agreement between the two $\mathrm{C} / \mathrm{O}$ core compositions found, the latter coming from evolutionary model being 43-57 \%. We find slightly more carbon in our homogeneous composition, but, all in all, it agrees nicely and boost our confidence in the proper sampling of the core. 


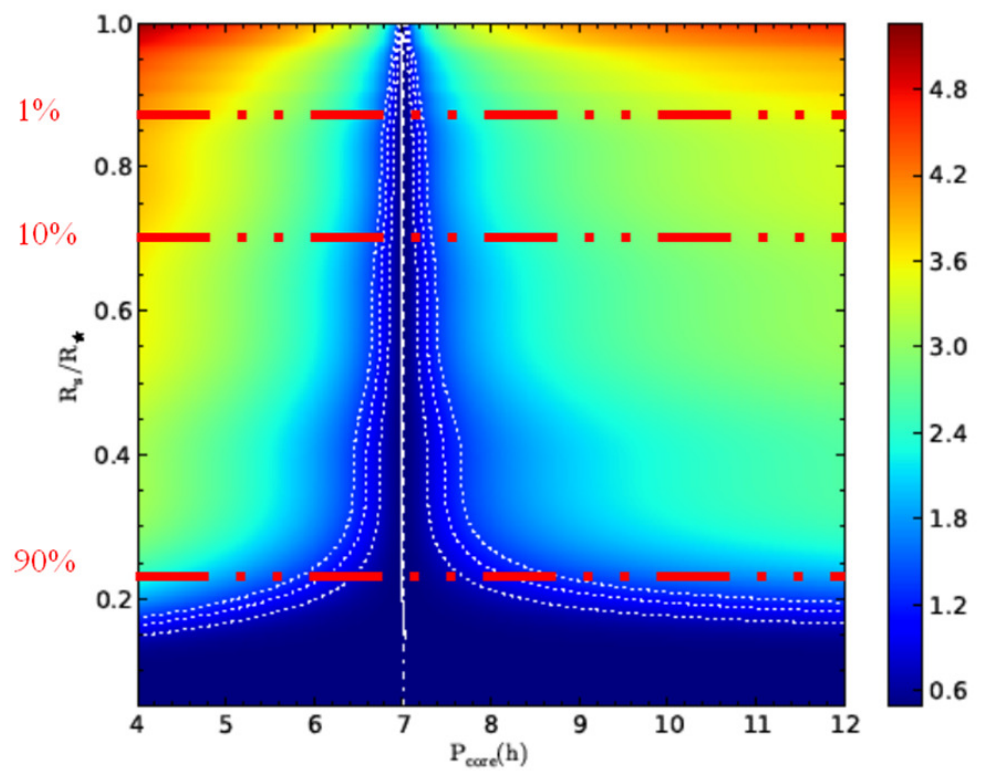

Fig. 4. Contour map showing the behaviour of the normalized merit function $S^{2}$ in terms of the radius and in terms of the rotation period of the inner region for solid body rotation in a two-zone approach defined by [1]. Horizontal dot-dashed curves give an indication on the encompassed mass. The solution is illustrated by the nearly vertical white curve and the dotted white curves depict the 1,2 and $3 \sigma$ contours. In comparison, the vertical dot-dashed white curve gives the exact solution for solid-body rotation. At larger depths, the gravity modes lose their ability to measure local rotation, giving the divergence in the 1,2 and $3 \sigma$ contours.

\section{Internal rotation profile}

Figure $3 \mathrm{~b}$ emphasizes the low-amplitude, but still present, rotational kernels in the interior of the star. Many rotationally-split structures sound the deep interior. Based on the optimal model of GD 1212, we determined its internal rotation profile as seen in Figure 4. Probing more than $85 \%$ of the radius, and more than $90 \%$ of the total mass, we can assert that GD 1212 is rotating as a solid body. Unlike other ZZ Ceti stars analysed, it is the first time that such a depth into the interior of a star of the kind is probed. Indeed, and unfortunately, "simple" blue edge pulsators analysed so far do not exhibit rotational splitting on modes present in the core of the star, and we can only sound a few percent of the radius into the interior.

\section{Conclusion}

These results boost our confidence in the reliability of the forward method with parameterized models for sounding white dwarf internal structure with asteroseismology. For the first time, we explore to great depths the internal composition and rotation profile of a ZZ Ceti star.

\section{References}

1. Charpinet, S.,Fontaine, G., \& Brassard, P., Nat 461, 501 (2009)

2. Fontaine, G., \& Brassard, P., PASP 120, 1043 (2008)

3. Greiss, S., Gänsicke, B. T., Hermes, J. J., et al., MNRAS 438, 3086 (2014)

4. Hermes, J. J., Charpinet, S., Barclay, T., et al., ApJ 789, 85 (2014) 